\title{
Calcium phosphate system for gene delivery: historical background and emerging opportunities
}

\author{
Babak Mostaghaci ${ }^{\S a}$, Brigitta Loretz ${ }^{* a}$, Claus-Michael Lehr ${ }^{* a, b}$ \\ ${ }^{a}$ Department of Drug Delivery (DDEL), Helmholtz-Institute for Pharmaceutical Research Saarland (HIPS), Helmholtz \\ Centre for Infection Research (HZI), Saarland University, Campus E8.1, 66123 Saarbrücken, Germany; ${ }^{b}$ Saarland \\ University, Department of Pharmacy, Saarbrücken, Germany.
}

\begin{abstract}
Calcium phosphate system has been used widely in in vitro gene delivery for almost four decades. Excellent biocompatibility and simple application have motivated the researchers to always consider this system in their transfection experiments. However, there was a major drawback regarding the low transfection efficiency of calcium phosphate. Hence, there have been many efforts in order to increase the gene delivery potential of this system. In this paper, the application of calcium phosphate in gene delivery is introduced. Moreover, the recent progresses in the application of calcium phosphate in the delivery of (oligo)nucleotides and different approaches to improve the properties of this system are reviewed.
\end{abstract}

Keywords: Calcium phosphate, hydroxyapatite, bioceramics, gene delivery, transfection, biocompatibility.

\section{INTRODUCTION}

Almost four decades back, calcium phosphate system was introduced to be used as in vitro gene delivery carrier. Many studies have been conducted regarding applications of these systems in delivery of (oligo)nucleotides to different types of eukaryotic cells. Despite all of the limitations, there are still big motivations for using this materials in the delivery of (oligo)nucleotides. The proper biocompatibility of calcium phosphates, easy preparation methods, and some intrinsic characteristics of calcium phosphates which increase their transfection potential are some of the properties which make this material a suitable system for transfection and rarely could be seen in other materials.

\section{CALCIUM PHOSPHATE}

The work of Graham and Van Der Eb which was published in 1973 demonstrated the first application of calcium phosphate as gene delivery carrier. They showed that this bioceramic can condense DNA and increase the transfection efficiency, just after performing few simple steps [1]. This first research initiated the vast application of this technology in in vitro gene delivery. But if we want to realize why calcium phosphate has become so popular, first we should have a better understanding of its structure and properties.

\footnotetext{
*Corresponding authors: Prof. Claus-Michal Lehr, Tel: +49-681-988061000, Fax: +49-681-98806-1009; E-mail: Claus-Michael.Lehr@helmholtzhzi.de; Dr. Brigitta Loretz, Tel: +49-681- 98806-1030, E-mail: Brigitta.Loretz@helmholtz-hzi.de

$\S_{\text {Current address: Physical Intelligence Department, Max-Planck-Institute }}$ for Intelligent Systems, Stuttgart, Germany
}

\subsection{Calcium Phosphates Family}

Calcium phosphate-based bioceramics have been used in medicine and dentistry since many years back. Applications include coating of dental implants [2-4], periodontal treatment [5-7], alveolar ridge augmentation [8-10], orthopedics [11-13], maxillofacial surgery and tissue engineering [14-16], and treatment of spinal injuries [17-19].

Bone is a natural nano-composite which is composed of organic and inorganic components. The inorganic part of bone is made of biological apatites, which provides mechanical strength and act as a repository for calcium, phosphorus, sodium, and magnesium. Biological apatite is structurally similar, though not fully identical, to the mineral hydroxyapatite $\left(\mathrm{HaP}, \mathrm{Ca}_{10}\left(\mathrm{PO}_{4}\right)_{6}(\mathrm{OH})_{2}\right)$. Hydroxyapatite is the most ubiquitous and well-known crystal structure among all members of calcium phosphate family. The most normal chemical composition of hydroxyapatite has a $\mathrm{Ca} / \mathrm{P}$ ratio of 1.67 [20]. In biomedical applications, different crystal phases of calcium phosphate are being used depending on the desired properties such as bioactivity or solubility. The stable phases of calcium phosphate are synthesized by changing the temperature and/or water content [21].

\subsection{Calcium Phosphate Nanoparticles}

Calcium phosphate particles with smaller size can show especial properties which expand their application range. Calcium phosphate nanoparticles with a size around $100 \mathrm{~nm}$ have a proper biocompatibility. Moreover, these particles have the capability to penetrate into the membrane of eukaryotic cells and bacteria. Because of such characteristics, calcium phosphate nanoparticles can be used in different biomedicine branches such as drug delivery, 
gene delivery, and imaging [22]. To produce high quality $\mathrm{HaP}$ bioceramic which is used to build artificial bone substitutes, using smaller particles is usually a better choice in front of bigger particles. Using $\mathrm{HaP}$ powder with a size in nanometric range results in a high quality casting and sintering procedures which leads to better biological and mechanical properties of the final biomedical construct [23].

\section{CALCIUM PHOSPHATE AS GENE DELIVERY CARRIER}

\subsection{Historical Background}

The first application of calcium phosphate in gene delivery was reported by Graham and Van Der EB in 1973. In their study, calcium phosphate precipitates were used for transfecting cells with Adenovirus 5 DNA to investigate their infectivity [1]. They diluted Adenovirus 5 DNA in phosphate buffer and after adding calcium chloride, the mixture was incubated with KB Cells. It was shown that by adding the calcium precursor, the uptake of DNA significantly increased which led to a higher infectivity. This technique showed a transfection efficiency 100 times more than the DEAE-dextran method which was a conventional protocol for in vitro gene delivery at that time.

Using the same approach, this group conducted another study to transform rat kidney cells with the DNA of human adenovirus 5. They clearly saw the round and densely packed cells which are the typical characteristics for the adenovirus transfection. They indicated that the "calcium technique" was a very efficient and reproducible method for adenovirus DNA transformation [24].

Graham, Veldhuisen and Wilkie used "calcium phosphate" method to investigate the infectivity of herpes simplex virus type I (HSV-I) [25]. In another study, Abrahams and Van Der EB transformed the cells from rat kidney cells and also mouse 3T3 cells with Simian Virus 40 DNA. The results showed the reproducibility of this method for in vitro transformation [26]. Calcium phosphate was also used successfully to transform primary baby rat kidney (BRK) cells with specific fragments of human adenoviruses 2 and 5 DNAs [27].

In 1976, Stow and Wilkie reported that treatment of cells with dimethyl sulphoxide (DMSO) after transforming them with "Herpes Simplex Virus DNA"/calcium phosphate complex increased the number of obtained plaques which was an indicator for more efficient transformation. In other conventional method for transformation (DEAE-dextran) using DMSO did not lead to significant enhancement [28].

Calcium phosphate method had become a common protocol for in vitro gene delivery in the 1980s. In 1981, Corsaro and Pearson investigated some of the parameters which affect the transformation efficiency, such as rinsing the cells with DMSO [29]. An additional variable in this experiment was investigated which was the exposure time of DNA/calcium phosphate complex to cells. It was shown that when a suboptimal DNA exposure time was used (e.g. 4-12 hours) the rinsing with DMSO increased the transformation efficiency. On the other hand, rinsing with DMSO had no effect when optimal conditions were applied. The results indicated that rinsing with DMSO did not have significant effect on transformation efficiency. Another result of their study was about the formation of calcium phosphate precipitates. It was shown that it would be better to add the DNA/ $\mathrm{CaCl}_{2}$ solution to the HEPES-phosphate buffer rather than in the reverse order. It was also concluded that it is important to mix the solutions in a drop-wise manner. [29].

In 1982, a research group at Yale University conducted a research on uptake mechanism of DNA/calcium phosphate complex into mammalian cells by using electron microscopy and fluorescent staining [30, 31]. Electron microscopy and filter hybridization studies revealed that most of the DNA enters into the cells by phagocytosis. The effect of different drugs and respiratory inhibitors on the uptake of DNA was also investigated. Results showed that the phagocytosis of the DNA is inhibited by respiratory inhibitors, such as Colcemid, which disassembles the microtubules. It was concluded that the uptake of DNA/calcium phosphate is through a "receptor mediated" phagocytosis mechanism. In another part of the study, it was shown that ATP-depleted and cold-treated cells were not able to adsorb the complex. Thus the uptake of DNA/calcium phosphate complex is an energy- and temperature-dependent process [30].

The $\mathrm{pH}$ of calcium phosphate forming solution has an important effect on cell transformation efficiency. It was shown that only when the calcium phosphate/DNA complex formation was conducted in the $\mathrm{pH}$ range of 7.1 to 7.5 , the cell uptake of the complex happened. In the case of $\mathrm{pH}$ above 7.5 , it was not possible to detect any cell uptake. The DNA/calcium phosphate ratio is also important regarding the uptake of the complexes. In the case of higher ratios (increase amount of DNA), the expression of cytoplasmic fluorescence was drastically reduced which was a sign of less efficient transformation [31].

One of the limitations in calcium phosphate system is due to the degradation of the DNA before arriving the nucleus, where gene expression takes place. In 1983, Luthman and Magnusson conducted a research on increasing the efficiency of transfection by inhibiting the lysosomal degradation of the DNA, using Chloroquine as a lysosomotropic compound. They went through a conventional calcium phosphate transformation procedure, but they added Chloroquine to the growth medium. It was shown that using Chloroquine can increase the number of transfected cells. This conclusion was supported by the results of experiments in which cells were transfected with linear forms of viral DNA. In that case, in Chloroquine treated cells, the number of DNA molecules which re-circularized and were able to replicate was much higher than in untreated cells [32].

Having the same idea, in 1984 a research group in Norway used different chemical agents to inhibit the intracellular degradation (such as 3-methyl adenine, $\mathrm{NH}_{4} \mathrm{Cl}$, FCPP, and etc.). It was shown that using the degradation inhibitors led to a higher transformation efficiency due to the increasing of the cytoplasmic level of exogenous DNA [33]. 
In 1987, a new method for gene delivery with calcium phosphate system was introduced. The new concept was the gradual formation of DNA/calcium phosphate complexes in the cell culture medium during the incubation with the cells. It was shown that the crucial factors that affect the transfection efficiency were the $\mathrm{pH}$ of the buffer which was used for calcium phosphate precipitation (optimized $\mathrm{pH}$ was 6.95) and the $\mathrm{CO}_{2}$ level during the incubation of the DNA with the cells. The amount and the form of DNA were shown to be important factors. The circular DNA can lead to a better transfection efficiency than the linear DNA, however the reason for this phenomenon was not clear at that time. This research indicated that the efficiency of this new transfection method was comparable to the efficiency of other common transfection systems (at the time of publication) [34].

In 1990, Orrantia and Chang investigated the intracellular distribution of DNA after the DNA/calcium phosphate complexes transported into the cells. Results showed that only a small fraction of internalized DNA could be found in the nucleus, where is the target for gene delivery. Investigating the nucleus materials revealed that the mouse cells retained $6.4 \%$ of internalized DNA while the human cells retained only $2.2 \%$. It was realized that transfection with DNA/calcium phosphate has not high efficiency, partly because most of the endocytosed DNA is quickly degraded in the nucleus and excreted to the cytosol [35].

In 1994, O'Mahoney and Adams modified the transfection procedure introduced by Chen and Okayama in 1987 and indicated that they came up with a reliable and reproducible method with high transfection efficiency. They showed that the critical factor in their method is the preincubation time of the DNA/ $\mathrm{CaCl}_{2} / \mathrm{BES}$-buffered saline prior to addition to the cells. Using the optimum condition in their research led to $100 \%$ efficiency [36].

In 1996, a research was conducted on electrochemical properties of DNA/calcium phosphate complexes. The study focused on changing the zeta potential as a function of altering the $\mathrm{pH}$ of calcium phosphate and DNA/calcium phosphate precipitates. For calcium phosphate, the point of zero charge $(p z c)$ and isoelectric point (iep) were found to be at $\mathrm{pH} 7.09$ and 7.0, respectively. By adding the plasmid DNA, both $p z c$ and iep points shifted to higher values of 7.18 and 7.15 [37].

In a forthcoming paper, it was revealed that the $\mathrm{pH}$ for the synthesis of DNA/calcium phosphate complexes and the concentration of DNA were the crucial factors which modulate the cell uptake. The results showed that optimum transfection efficiency occurred in the $\mathrm{pH}$ range close to the iep of DNA/calcium phosphate precipitates ( $\mathrm{pH} 7.15)$. The zeta potentials of the calcium phosphate/DNA precipitates which were synthesized in the absence of DMEM and calf serum were determined to be in the range of 11 to $21 \mathrm{mV}$. Synthesis within these limits resulted in an efficient uptake of the DNA/calcium phosphate complexes [38].

In 2004, Jordan and Wurm conducted a survey on the protocols which were used previously for gene delivery with calcium phosphate particles. They stated that all different protocols which were mentioned in the literature are based on the same principle: a spontaneous precipitation of calcium phosphate which occurs in supersaturated solutions. Although a wide range of conditions can lead to the precipitation of calcium phosphate, high transfection efficiency is only obtained within a narrow range of optimized parameters [39].

\subsection{Current Studies}

Nanotechnology made new opportunities for the application of calcium phosphate in gene delivery. Studies have been conducted to show the feasibility of using calcium phosphate, in nano-metric particulate form, in gene delivery and optimizing the parameters.

In the University of Duisburg-Essen, multi-shell calcium phosphate/DNA particles were prepared. It was shown that by using multi-shell calcium phosphate/DNA nanoparticles the transfection efficiency was increased due to the protection of DNA against nuclease enzymes. The authors indicated that in contrast to conventional calcium phosphate, these particles could be stored for weeks without losing their transfection efficiency [40]. One of the limitations of conventional calcium phosphate transfection method is unbalancing of the intracellular calcium homeostasis. With the new multi-shell nanoparticles, it was shown that the intracellular calcium level remained in the standard range after transfection. This positive behavior can be an indication for a higher biocompatibility of these particles [41].

Hanifi et al. conducted research on the feasibility of using strontium and magnesium substituted calcium phosphate in gene delivery applications. A simple sol-gel method was recruited in their research. They obtained nanostructured calcium phosphate particles with a high specific surface area, and a high dissolution rate. The zeta potential was increased in comparison to non-doped calcium phosphate particles. It was indicated that due to the increased surface charge and solubility, these new system can increase the transfection efficiency [42, 43]. The multi-shell calcium phosphate carrying the pDNA encoded BMP-7 or VEGF-A proteins was also used to develop injectable cement for bone repair [44].

Recently there have been studies on using calcium phosphate in mixture with other materials in order to improve its capabilities as a gene delivery carrier. Stabilizing with bis-phosphonate [45] or glutamine-chitosan [46], coating with lipids [47-49], and association with Adenovirus [50-53] are some examples for this approach. In our recent study we functionalized the surface of calcium phosphate nanoparticles with aminosilane. We showed that aminosilane functionalization can change the $\mathrm{pH}$ boundary between two crystalline phases of calcium phosphate: brushite and HaP. We conducted gene delivery experiments using both types of nanoparticles and realized that using brushite nanoparticles led to a higher transfection efficiency, comparable to a commercial in vitro transfection reagent (Figure 1). These particles were biocompatible and did not show significant adverse toxic or immunogenic effects $[54,55]$. 
Coupling of calcium phosphate with other polymers (sometimes cationic polymers) is another solution to use this bioceramic in gene delivery. Polyethylene glycol showed the capability to speed up the endosomal escape and as a result increase the transfection efficiency [56]. PLGA is another polymer which coupled with calcium phosphate in order to enhance its gene delivery capabilities [57, 58]. Linear polyethylene amine (PEI) was used to coat the calcium phosphate particles containing microRNA. More homogenous particles were produced due to the charge of the particles. Moreover, the results showed a significant increase in transfection efficiency [59]. Branched PEI was also used to modify the surface of hydroxyapatite nanoparticles used for siRNA delivery. The results indicated that the transfection efficiency was comparable with the commercial transfection reagents [60].
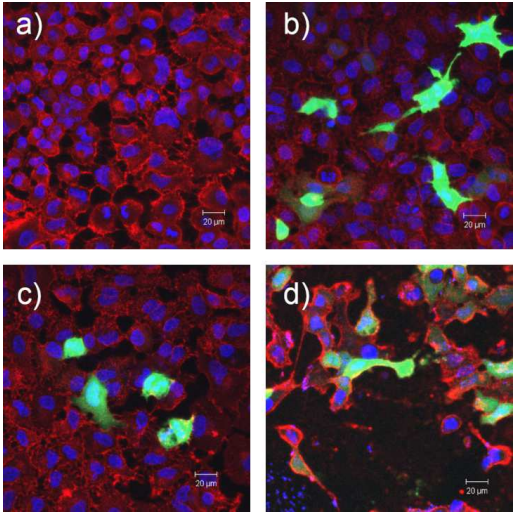

Figure 1: CLSM images of a) untreated A549 cells, b-d) A549 cells transfected with pAmCyan using b) modified Brushite NPs, c) modified HaP NPs, d) jetPRIME transfection reagent. Green: Fluorescent Protein, Blue: Cell nuclei, Red: Cell membrane. (Reprinted from [55] with the permission from ACS)

In order to increase the transfection efficiency or having more control on the movement of calcium phosphate gene delivery carriers, some groups coupled calcium phosphate with magnetic materials. Puddu et al. designed iron oxide doped tri calcium phosphate nanoparticles. They showed that it is possible to concentrate the particles and the bound pDNA in a specific point in the cell culture plate by applying the magnetic field. They did not observe a significant toxicity but they got localized increasing in gene transfection [61]. Other groups also reported this approach using different structures of calcium phosphate and different types of magnetic materials [62, 63].

\subsection{In vivo Studies}

There has been some efforts to use calcium phosphate for in vivo gene delivery. Because of the structural similarities, bone tissue is always considered as the best target for calcium phosphate to deliver drug or oligonucleotides.
However, some studies also conducted in order to use calcium phosphate as a systemic gene delivery carrier. Here, we briefly review the recent in vivo applications of calcium phosphate-based gene delivery carriers.

Localized gene therapy for treatment of bone defects is one of the main applications of in vivo gene delivery with calcium phosphate. Krebs et al. developed alginate-based injectable scaffold contained calcium phosphate nanoparticles which condensed a pDNA encoding an osteoconductive protein (BMP-2). Using mice models, they showed that it is possible to have an efficient bone regeneration using this method [64]. In another study a tissue engineering scaffold made of collagen and calcium phosphate was used to stimulate bone formation by transfecting cells with VEGF pDNA. It was shown that this system can be used for localized gene delivery without the need for additional gene delivery vector. Sustained release of pDNA was an important advantage for this system [65].

Other than bone, using calcium phosphate for gene delivery to other tissues have been also investigated. In one study, the application of PEGylated lipid-coated calcium phosphate nanoparticles for systemic delivery of siRNA was investigated. The results showed a very good bio-distribution of the labeled siRNA [66]. Using the same approach, in another study the accumulation of lipid/calcium phosphate nanoparticles in the cancerous lymph nodes were observed after intravenous injection of these particles. The system was carrying a fluorescent cDNA which expressed in the lymph nodes [67]. Lee et al. stabilized calcium phosphate nanoparticles with a conjugate of dopa and hyaluronic acid and used these particles for systemic delivery of siRNA. Higher intratumoral accumulation of siRNA and improved gene silencing were observed as a result of this approach [68].

\section{CONCLUSION}

In biomedical engineering, calcium phosphate has an especial place because of its biocompatibility and easy preparation procedure. The capability of this material for DNA condensation makes it a potential choice as gene delivery carrier. Calcium phosphate nanoparticles are able to condense DNA and take it into the target cells. This system is recognized as one of the highly appealing systems for in vitro gene delivery.

Calcium phosphate system still have some problems which should be considered in future studies. If calcium phosphate/DNA complex degraded in the blood circuit, it could not be used in most of the clinical gene delivery applications. The other problem is the low transfection efficiency of calcium phosphate system, which currently limits the application of this system. Different transfection efficiencies for calcium phosphate system have been reported which is mostly due to the instability of calcium phosphate in aqueous solutions. Once these problems are overcome, the excellent biocompatibility and biodegradability of calcium phosphate could lead to more applications of this system in vitro and in vivo. 


\section{REFERENCES}

[1] Graham FL and Van Der EB AJ. New Technique for Assay of Infectivity of Human Adenovirus 5 DNA. Virology 1973. 52(2): 456-467.

[2] Pardun K, et al. Mixed zirconia calcium phosphate coatings for dental implants: Tailoring coating stability and bioactivity potential. Mat Sci Eng C-Mater 2015. 48(337-346

[3] Yokota S, et al. Evaluation of Thin Amorphous Calcium Phosphate Coatings on Titanium Dental Implants Deposited Using Magnetron Sputtering. Implant Dent 2014. 23(3): 343-350.

[4] Kim S, Jung UW, Lee YK, and Choi SH. The effect of calcium phosphate bone substitute on defect resolution around a roughsurfaced dental implants in dogs. J Biomed Mater Res B 2011. 99B(1): 21-26.

[5] Pietruska M, et al. Four-year results following treatment of intrabony periodontal defects with an enamel matrix derivative alone or combined with a biphasic calcium phosphate. Clin Oral Invest 2012. 16(4): 1191-1197.

[6] Lee MJ, Kim BO, and Yu SJ. Clinical evaluation of a biphasic calcium phosphate grafting material in the treatment of human periodontal intrabony defects. J Periodontal Implan 2012. 42(4): 127-135.

[7] Struillou X, et al. Treatment of periodontal defects in dogs using an injectable composite hydrogel/biphasic calcium phosphate. J Mater Sci-Mater M 2011.22(7): 1707-1717.

[8] Friedmann A, Gissel K, Konermann A, and Gotz W. Tissue reactions after simultaneous alveolar ridge augmentation with biphasic calcium phosphate and implant insertion-histological and immunohistochemical evaluation in humans. Clin Oral Invest 2015. 19(7): 1595-1603.

[9] Sato I, et al. Histological evaluation of alveolar ridge augmentation using injectable calcium phosphate bone cement in dogs. J Oral Rehabil 2009. 36(10): 762-769.

[10] Sugawara A, et al. Histopathologic reaction of a calcium phosphate cement for alveolar ridge augmentation. J Biomed Mater Res 2002. 61(1): 47-52

[11] Begam H, Mandal S, Chanda A, Mukherjee J, and Nandi SK. Effect of Zinc Doping on Biological Properties of Biphasic Calcium Phosphate Ceramics in Orthopaedic Animal Model. T Indian Ceram Soc 2014. 73(4): 284-292.

[12] Palaniappan N, Inwati GK, and Singh M. Biomaterial Co-Cr-Mo Alloys Nano Coating Calcium Phosphate Orthopedic Treatment. Iop Conf Ser-Mat Sci 2014. 64(

[13] Van der Stok J, et al. Properties of commonly used calcium phosphate cements in trauma and orthopaedic surgery. Injury 2013. 44(10): 1368-1374.

[14] Eppley B. Development and clinical results of calcium phosphate materials for cranial and maxillofacial surgery. Rev Chir Orthop 2000. 86(5): 520-521.

[15] Klammert U, et al. 3D powder printed calcium phosphate implants for reconstruction of cranial and maxillofacial defects. J Cranio Maxill Surg 2010. 38(8): 565-570.

[16] Urkmez AS, Clark SG, Wheeler MB, Goldwasser MS, and Jamison RD. Evaluation of chitosan/biphasic calcium phosphate scaffolds for maxillofacial bone tissue engineering. Macromol Symp 2008. 269(100-105.

[17] Nagineni VV, et al. Silicate-Substituted Calcium Phosphate Ceramic Bone Graft Replacement for Spinal Fusion Procedures. Spine 2012. 37(20): E1264-E1272.

[18] Majid K, Tseng MD, Baker KC, Reyes-Trocchia A, and Herkowitz HN. Biomimetic calcium phosphate coatings as bone morphogenetic protein delivery systems in spinal fusion. Spine J 2011. 11(6): 560-567.

[19] Hui CFF, et al. Low-Intensity Pulsed Ultrasound Enhances Posterior Spinal Fusion Implanted with Mesenchymal Stem Cells-Calcium
Phosphate Composite Without Bone Grafting. Spine 2011. 36(13): 1010-1016.

[20] Narayan RJ, et al. Nanostructured ceramics in medical devices: Applications and prospects. Jom-Us 2004. 56(10): 38-43.

[21] Hench LL. Bioceramics - from Concept to Clinic. J Am Ceram Soc 1991. 74(7): 1487-1510.

[22] Epple M, et al. Application of calcium phosphate nanoparticles in biomedicine. J Mater Chem 2010. 20(1): 18-23.

[23] Cao LY, Zhang CB, and Huang HF. Synthesis of hydroxyapatite nanoparticles in ultrasonic precipitation. Ceramics International 2005. 31(8): 1041-1044.

[24] Graham FL and Van Der EB AJ. Transformation of Rat Cells by DNA of Human Adenovirus-5. Virology 1973. 54(2): 536-539.

[25] Graham FL, Veldhuis.G, and Wilkie NM. Infectious Herpesvirus DNA. Nature-New Biol 1973. 245(148): 265-266.

[26] Abrahams PJ and Van Der Eb AJ. In vitro transformation of rat and mouse cells by DNA from simian virus 40. J Virol 1975. 16(1): 206-9.

[27] Van Der EB AJ, Mulder C, Graham FL, and Houweling A. Transformation with Specific Fragments of Adenovirus Dnas .1. Isolation of Specific Fragments with Transforming Activity of Adenovirus-2 and 5 DNA. Gene 1977. 2(3-4): 115-132.

[28] Stow ND and Wilkie NM. Improved Technique for Obtaining Enhanced Infectivity with Herpes-Simplex Virus Type-1 DNA. J Gen Virol 1976. 33(Dec): 447-458.

[29] Corsaro CM and Pearson ML. Enhancing the Efficiency of DNAMediated Gene-Transfer in Mammalian-Cells. Somat Cell Genet 1981. 7(5): 603-616.

[30] Loyter A, Scangos G, Juricek D, Keene D, and Ruddle FH Mechanisms of DNA Entry into Mammalian-Cells .2. Phagocytosis of Calcium-Phosphate DNA Coprecipitate Visualized by Electron-Microscopy. Exp Cell Res 1982. 139(1): 223-234.

[31] Loyter A, Scangos GA, and Ruddle FH. Mechanisms of DNA Uptake by Mammalian-Cells - Fate of Exogenously Added DNA Monitored by the Use of Fluorescent Dyes. P Natl Acad Sci-Biol 1982. 79(2): 422-426.

[32] Luthman $\mathrm{H}$ and Magnusson G. High-Efficiency Polyoma DNA Transfection of Chloroquine Treated-Cells. Nucleic Acids Res 1983. 11(5): 1295-1308.

[33] Ege T, Reisbig RR, and Rogne S. Enhancement of DNA-Mediated Gene-Transfer by Inhibitors of Autophagic-Lysosomal Function. Exp Cell Res 1984. 155(1): 9-16.

[34] Chen C and Okayama H. High-Efficiency Transformation of Mammalian-Cells by Plasmid DNA. Mol Cell Biol 1987. 7(8): 2745-2752.

[35] Orrantia E and Chang PL. Intracellular-Distribution of DNA Internalized through Calcium-Phosphate Precipitation. Exp Cell Res 1990. 190(2): 170-174.

[36] Omahoney JV and Adams TE. Optimization of ExperimentalVariables Influencing Reporter Gene-Expression in HepatomaCells Following Calcium-Phosphate Transfection. DNA and cell biology 1994. 13(12): 1227-1232.

[37] Yang YW and Yang JC. Characterization of Calcium Phosphate as a Gene Carrier (I): Electrochemical Properties. Drug Delivery 1996. 3(173-179.

[38] Yang YW and Yang JC. Characterization of Calcium Phosphate as a Gene Carrier (II): Zeta Potential and DNA Transfection. Drug Delivery 1996. 3(181-186.

[39] Jordan M and Wurm F. Transfection of adherent and suspended cells by calcium phosphate. Methods 2004. 33(2): 136-143.

[40] Sokolova VV, Radtke I, Heumann R, and Epple M. Effective transfection of cells with multi-shell calcium phosphate-DNA nanoparticles. Biomaterials 2006. 27(16): 3147-53.

[41] Neumann S, Kovtun A, Dietzel ID, Epple M, and Heumann R. The use of size-defined DNA-functionalized calcium phosphate 
nanoparticles to minimise intracellular calcium disturbance during transfection. Biomaterials 2009. 30(35): 6794-802.

[42] Hanifi A, Fathi MH, and Sadeghi HMM. Effect of strontium ions substitution on gene delivery related properties of calcium phosphate nanoparticles. J Mater Sci-Mater M 2010. 21(9): 2601-2609.

[43] Hanifi A, Fathi MH, Sadeghi HMM, and Varshosaz J. Mg2+ substituted calcium phosphate nano particles synthesis for non viral gene delivery application. J Mater Sci-Mater M 2010. 21(8): 2393-2401

[44] Chernousova S, Klesing J, Soklakova N, and Epple M. A genetically active nano-calcium phosphate paste for bone substitution, encoding the formation of BMP-7 and VEGF-A. Rsc Adv 2013. 3(28): 11155-11161.

[45] Giger EV, et al. Gene delivery with bisphosphonate-stabilized calcium phosphate nanoparticles. Journal of controlled release : official journal of the Controlled Release Society 2011. 150(1): 87-93.

[46] Choi BY, et al. Glutamine-chitosan modified calcium phosphate nanoparticles for efficient siRNA delivery and osteogenic differentiation. J Mater Chem B 2015. 3(31): 6448-6455.

[47] Zhou C, et al. Lipid-coated nano-calcium-phosphate (LNCP) for gene delivery. International journal of pharmaceutics 2010. 392(1-2): 201-8.

[48] Tang J, et al. Preparation of optimized lipid-coated calcium phosphate nanoparticles for enhanced in vitro gene delivery to breast cancer cells. J Mater Chem B 2015. 3(33): 6805-6812.

[49] Haynes MT and Huang L. Lipid-Coated Calcium Phosphate Nanoparticles for Nonviral Gene Therapy. Adv Genet 2014. 88(205-229.

[50] Toyoda K, Andresen JJ, Zabner J, Faraci FM, and Heistad DD. Calcium phosphate precipitates augment adenovirus-mediated gene transfer to blood vessels in vitro and in vivo. Gene Ther 2000. 7(15): 1284-91.

[51] Feng L, et al. Optimization of transfection mediated by calcium phosphate for plasmid rAAV-LacZ (recombinant adenoassociated virus-beta-galactosidase reporter gene) production in suspension-cultured HEK-293 (human embryonic kidney 293) cells. Biotechnol Appl Bioc 2007. 46(127-135.

[52] Yang YW and Chao CK. Incorporation of calcium phosphate enhances recombinant adeno-associated virus-mediated gene therapy in diabetic mice. J Gene Med 2003. 5(5): 417-424.

[53] Walters RW, Duan D, Engelhardt JF, and Welsh MJ. Incorporation of adeno-associated virus in a calcium phosphate coprecipitate improves gene transfer to airway epithelia in vitro and in vivo. $\mathrm{J}$ Virol 2000. 74(1): 535-540

[54] Mostaghaci B, Susewind J, Kickelbick G, Lehr CM, and Loretz B. Transfection system of amino-functionalized calcium phosphate nanoparticles: in vitro efficacy, biodegradability, and immunogenicity study. ACS applied materials \& interfaces 2015. 7(9): 5124-33.

[55] Mostaghaci B, Loretz B, Haberkorn R, Kickelbick G, and Lehr CM. One-Step Synthesis of Nanosized and Stable AminoFunctionalized Calcium Phosphate Particles for DNA Transfection. Chem Mater 2013. 25(18): 3667-3674.
[56] Pittella F, et al. Enhanced endosomal escape of siRNA-incorporating hybrid nanoparticles from calcium phosphate and PEG-block charge-conversional polymer for efficient gene knockdown with negligible cytotoxicity. Biomaterials 2011. 32(11): 3106-14.

[57] Tang J, et al. Calcium phosphate embedded PLGA nanoparticles: a promising gene delivery vector with high gene loading and transfection efficiency. International journal of pharmaceutics 2012. 431(1-2): 210-21.

[58] Wang KW, et al. Calcium phosphate/PLGA-mPEG hybrid porous nanospheres: A promising vector with ultrahigh gene loading and transfection efficiency. J Mater Chem 2010. 20(6): 1161-1166.

[59] Jung H, et al. Long chain microRNA conjugates in calcium phosphate nanoparticles for efficient formulation and delivery. Arch Pharm Res 2015. 38(5): 705-715.

[60] Xu XL, et al. Hydroxyapatite nanoparticles modified by branched polyethylenimine are effective non-viral vectors for siRNA transfection of hepatoma cells in vitro. Int J Oncol 2015. 46(5): 2138-2142.

[61] Puddu M, et al. Magnetically deliverable calcium phosphate nanoparticles for localized gene expression. Rsc Adv 2015. 5(13): 9997-10004.

[62] Zuo GF, Wan YZ, and Zhang Y. Preparation and characterization of a novel laminated magnetic hydroxyapatite for application on gene delivery. Mater Lett 2012. 68(225-227.

[63] Wu HC, Wang TW, Bohn MC, Lin FH, and Spector M. Novel Magnetic Hydroxyapatite Nanoparticles as Non-Viral Vectors for the Glial Cell Line-Derived Neurotrophic Factor Gene. Adv Funct Mater 2010. 20(1): 67-77.

[64] Krebs MD, Salter E, Chen E, Sutter KA, and Alsberg E. Calcium phosphate-DNA nanoparticle gene delivery from alginate hydrogels induces in vivo osteogenesis. Journal of biomedical materials research. Part A 2010. 92(3): 1131-8.

[65] Keeney M, van den Beucken JJ, van der Kraan PM, Jansen JA, and Pandit A. The ability of a collagen/calcium phosphate scaffold to act as its own vector for gene delivery and to promote bone formation via transfection with $\operatorname{VEGF}(165)$. Biomaterials 2010. 31(10): 2893-902.

[66] Li J, Yang Y, and Huang L. Calcium phosphate nanoparticles with an asymmetric lipid bilayer coating for siRNA delivery to the tumor. Journal of Controlled Release 2012. 158(1): 108-114.

[67] Tseng YC, Xu ZH, Guley K, Yuan H, and Huang L. Lipid-calcium phosphate nanoparticles for delivery to the lymphatic system and SPECT/CT imaging of lymph node metastases. Biomaterials 2014. 35(16): 4688-4698.

[68] Lee MS, et al. Target-specific delivery of siRNA by stabilized calcium phosphate nanoparticles using dopa-hyaluronic acid conjugate. Journal of controlled release : official journal of the Controlled Release Society 2014. 192(122-30.

Some parts of this work are adapted from "Mostaghaci et al., NanoParticulate Calcium Phosphate as a Gene Delivery System. In: Non-Viral Gene Therapy, Xubo Yuan (Ed.), InTech. (C) 2011 Babak Mostaghaci, Arash Hanifi, Brigitta Loretz \& Claus-Michael Lehr. Originally published under CC BY-NC-SA 3.0 license. Available from: DOI 10.5772/18948 